\title{
PRINCE Secondary: transdiagnostic CBT is not effective for persistent physical symptoms
}

PRINCE Secondary was a randomized trial to test the efficacy and cost-effectiveness of therapist-delivered, transdiagnostic cognitive behavioural therapy (TDT-CBT) for patients with persistent physical symptoms (PPS) (Chalder et al., 2021). 324 PPS patients were randomized to receive either TDT-CBT plus standard medical care (SMC) or SMC alone. The trial's primary outcome was the mean score on the Work and Social Adjustment Scale (WSAS) at follow-up assessment 52 weeks post-randomization. The trial also included several secondary outcomes.

In the conclusion of the abstract, the authors state that the trial provides "preliminary evidence that TDT-CBT + SMC may be helpful for people with a range of PPS." This statement is misleading since it ignores the null findings of the trial's primary outcome. Although the intervention group reported a modest benefit on the WSAS over the SMC group, it was not statistically or clinically significant. For the WSAS, the authors designated a reduction of -3.6. as a minimum clinically important difference (MCID). At 12 months, the mean for the intervention group was -1.48 points less than for the SMC group, with a 95 per cent confidence interval of 3.44 to 0.48 . The confidence interval thus excluded what the authors had predefined as the MCID. This indicates that the trial was adequately powered to provide strong and actionable evidence of efficacy but that it failed to do so.

Nonetheless the authors advance their argument of "preliminary evidence" that the intervention was "helpful" by reporting that "some secondary outcomes had a treatment effect in favour of TDT-CBT + SMC". Specifically, three out of the eight secondary outcomes listed in the paper had results that were statistically significant yet of questionable clinical value. One was the WSAS at 20 weeks where the mean score in the intervention arm was 2.41 points lower than in the control group. The authors emphasize this result because it reached statistical significance even though the reported benefit was less than the predefined MCID of -3.6. This assessment of the WSAS at 20 weeks was also not listed as a secondary outcome in either the trial protocol (Chalder et al., 2019) or the trial registration (NCT02426788).

Similarly, the authors highlight a statistically significant difference on another secondary outcome, the Patient Health Questionnaire 15 (PHQ15) assessed 52 weeks post-randomization. They did not provide an MCID for this scale, as they did for the WSAS. However, the difference between groups was -1.51 points on a 0-30 point scale, lower than MCID estimates for the PHQ15 proposed by other researchers (Claassen-van Dessel et al., 2018; Toussaint et al., 2017). The authors also highlight a statistically significant result on the Clinical Global Impression scale. Here the difference between control and intervention group was 0.55 points, lower than 1 point on the 1-9 Likert scale, in which each full point represents only a small change from the preceding one.

Even these exceedingly modest results, however, are open to question. The authors themselves indicate that the findings would not be statistically significant if adjusted for multiple comparisons by more conservative methods such as the Bonferroni correction. Moreover, the study relied solely on subjective outcomes even though neither patients nor therapists could be blinded to treatment allocation - a combination of factors likely to result in an unknown amount 
of response bias. Patients in the comparison group received no intervention, and the investigators did not control for the differences in contact time with healthcare professionals. Perhaps the extra attention and heightened expectations of trial participants in the intervention arm, rather than the nature of the intervention itself, influenced how they answered questionnaires. Unfortunately, these weaknesses in trial design were not discussed as limitations in the paper. Both complicate the interpretation of differences in favour of the TDT-CBT + SMC group.

No such interpretation problems arise, however, when the $95 \%$ confidence interval of the difference between groups excludes the MCID. By the trial's own metrics for assessing the success of the intervention, the core message to be drawn is that TDT-CBT was not effective at treating patients suffering from PPS. It is unfortunate that the authors do not make this clear. Those who only read the abstract will receive an inaccurate message.

\section{References}

Chalder, T., Patel, M., Hotopf, M., Moss-Morris, R., Ashworth, M., Watts, K., David, A. S., McCrone, P., Husain, M., Garrood, T., James, K., \& Landau, S. (2021). Efficacy of therapist-delivered transdiagnostic CBT for patients with persistent physical symptoms in secondary care: A randomised controlled trial. Psychological Medicine, 1-11. https://doi.org/10.1017/S0033291721001793

Chalder, T., Patel, M., James, K., Hotopf, M., Frank, P., Watts, K., McCrone, P., David, A., Ashworth, M., Husain, M., Garrood, T., Moss-Morris, R., \& Landau, S. (2019). Persistent physical symptoms reduction intervention: A system change and evaluation in secondary care (PRINCE secondary) - a CBTbased transdiagnostic approach: study protocol for a randomised controlled trial. BMC Psychiatry, 19(1), 307. https://doi.org/10.1186/s12888-019-2297-y

Claassen-van Dessel, N., van der Wouden, J. C., Hoekstra, T., Dekker, J., \& van der Horst, H. E. (2018). The 2-year course of Medically Unexplained Physical Symptoms (MUPS) in terms of symptom severity and functional status: Results of the PROSPECTS cohort study. Journal of Psychosomatic Research, 104, 76-87. https://doi.org/10.1016/j.jpsychores.2017.11.012

Toussaint, A., Kroenke, K., Baye, F., \& Lourens, S. (2017). Comparing the Patient Health Questionnaire - 15 and the Somatic Symptom Scale - 8 as measures of somatic symptom burden. Journal of Psychosomatic Research, 101, 44-50. https://doi.org/10.1016/j.jpsychores.2017.08.002 